\title{
miR-26b inhibits proliferation, migration, invasion and apoptosis induction via the downregulation of 6-phosphofructo-2-kinase/fructose-2,6-bisphosphatase-3 driven glycolysis in osteosarcoma cells
}

\author{
JING-YU DU, LI-FENG WANG, QUAN WANG and LIE-DAO YU \\ Department of Orthopedics, The First Affiliated Hospital of Zhejiang University, \\ Zhejiang University, Hangzhou, Zhejiang 310006, P.R. China
}

Received October 6, 2014; Accepted January 9, 2015

DOI: 10.3892/or.2015.3797

\begin{abstract}
MicroRNAs (miRNAs) are differentially expressed and play crucial roles in cancer development and progression. Elevated glycolysis provides survival advantage and metastatic phenotype. Emerging evidence indicates that glycolysis in cancers can be regulated by miRNAs. In the present study, the role of miR-26b in the proliferation, invasion and glycolytic phenotype of osteosarcoma (OS) cells was investigated. miR-26b was reported to be downregulated in OS tissues, however, the effect of miR-26b on OS has not been distinctly evaluated. The present study therefore investigated the miR-26b sensitivity mechanism in OS. To determine the role of miR-26, we reinstated its expression in the U2OS OS cell line through transfection with miR-26b mimics and examined the effects on cell proliferation, migration, invasion, cell cycle progression and glycolytic parameters. The computational prediction tool was employed to identify the molecular target of miR-26b and was confirmed experimentally. Restoration of miR-26b expression inhibited cell proliferation, migration and invasion, arrested cell cycle progression, and induced cell apoptosis accompanied by the downregulation of glycolytic phenotype. Moreover, the binding site for miR-26b was predicted in the 3'UTR of gene 6-phosphofructo-2-kinase/fructose-2,6-bisphosphatase-3 (PFKFB3), suggesting a role for miR-26b in metabolic alteration in OS cells. Further studies showed that overexpression of miR-26b repressed PFKFB3 mRNA and protein levels followed by modulation of the expression of glycolytic components (LDHA, GLUT-1) and markers of invasion and cell cycle such as MMP-9, MMP-2, cyclin D1 and
\end{abstract}

Correspondence to: Dr Lie-Dao Yu, Department of Orthopedics, The First Affiliated Hospital of Zhejiang University, Zhejiang University, No. 79 Qingchun Road, Hangzhou, Zhejiang 310006, P.R. China E-mail: liedaoyu@gmail.com

Key words: miR-26b, PFKFB3, glycolysis, proliferation, migration, invasion, osteosarcoma p27. Collectively, the data suggested the tumor suppressive role of miR-26b which functions by targeting the glycolytic metabolism in OS cells, and providing a possible therapeutic strategy for OS patients by targeting miRNA expression.

\section{Introduction}

Osteosarcoma (OS) is the most common primary bone tumor in children and young adults characterized by osteoid production and osteoblastic formation (1). Considerable improvement in the prognosis of patients has been observed due to the development of various adjuvant chemotherapies (2). However, the complete effectiveness of these chemotherapies is controversial as $80 \%$ of the patients would eventually developed metastatic disease. Thus, the outcome remains unsatisfactory for these patients (3). Therefore, the identification of effector molecules and signaling pathways that exhibits a close relationship with tumor progression and metastasis in order to improve the existing OS treatment, is crucial.

MicroRNAs (miRNAs) are small (22-24 nucleotides) non-coding RNAs emerging as promising diagnostic and prognostic tools of malignant neoplasm (4). miRNA is crucial in the regulation of diverse target mRNAs at the level of mRNA degradation or translation (5). It has been reported that biological activities of various miRNAs contribute to invasion and metastasis in OS (6). Emerging evidence indicates that miR-26b is downregulated in breast cancer (7), nasopharyngeal carcinoma (8), colorectal cancer (9), hepatocellular carcinoma (10) and in OS (6). However, the biological effect of miR-26b in OS tumorigenesis and metastasis remains to be elucidated. Therefore, it is of utmost significance to investigate the mechanism of miR-26b in OS.

Metabolic reprogramming accompanied by persistent aerobic glycolysis and deregulated mitochondrial function promotes OS progression and metastasis $(11,12)$. 6-Phosphofructo-2-kinase/fructose-2,6-bisphosphatases (PFKFBs) possess bifunctional enzymatic activities that regulate a high glycolytic flux by controling the intracellular concentration of fructose-2,6-bisphosphate (F2,6BP), a potent allosteric regulator of 6-phosphofructo-1-kinase (PFK-1) (13). PFKFB3 is one of the four isoforms of the PFKFB family, the protein 
expression of which is elevated in most types of tumor, including OS (14), suggesting its contribution to attaining glycolytic phenotype by several malignancies (11).

In the present study, we demonstrated that restoring the expression of miR-26b in the human U2OS OS cell line caused profound suppression of the proliferation, migration, invasion, cell cycle arrest and induction of apoptosis. Furthermore, computational prediction and experimental confirmation suggested that the tumor suppressive effects of miR-26b in OS cells were mediated through the inhibition of PFKFB3. Our results showed that an ectopic expression of miR-26b significantly suppressed the expression of PFKFB3 and glycolytic activity indicated by a decrease in extracellular lactate, ATP levels and glucose consumption. These results emphasized the antiproliferative and anti-metabolic role of miR-26b in OS cells and provided a basis to therapeutically intervene in metastasis of OS patients by targeting miRNA expression.

\section{Materials and methods}

Cell culture and transfection. Human U2OS OS cells were obtained from ATCC (Manassas, VA, USA) and cultured in Dulbecco's modified Eagle's medium (DMEM) supplemented with $10 \%$ heat-inactivated fetal bovine serum (FBS), streptomycin $(100 \mathrm{lg} / \mathrm{ml})$ and penicillin $(100 \mathrm{U} / \mathrm{ml})$ (Sigma-Aldrich, St. Louis, MO, USA). Cell cultures were maintained in $5 \% \mathrm{CO}_{2}$ atmosphere at $37^{\circ} \mathrm{C}$ in a humidified incubator. The hsa-miR-26b mimics (Pre-miR miRNA Precursor Product, AM17100) and its scramble mimics (Pre-miR Negative Control, AM17111) were purchased from Applied Biosystems (Foster City, CA, USA). PFKFB3 and control siRNA were purchased from Dharmacon (Austin, TX, USA). Transfection of the oligonucleotides was performed using Lipofectamine 2000 (Invitrogen, Carlsbad, CA, USA) according to the manufacturer's instructions. After $6 \mathrm{~h}$ the original medium was replaced with fresh medium.

Cell proliferation assay. The cell proliferation assay was determined by Cell Counting Kit- 8 assay (Dojindo, Kumamoto, Japan), a redox assay similar to 3-(4,5-dimethylthiazol2-yl)-2,5-diphenyltetrazolium bromide (MTT) according to the manufacturer's instructions. The proliferation level was determined at $0,24,48,72$ and $96 \mathrm{~h}$ after transfection. CCK-8 solution $(10 \mu \mathrm{l})$ was added to each well, followed by incubation for $2 \mathrm{~h}$ at $37^{\circ} \mathrm{C}$. The absorbance at $450 \mathrm{~nm}$ was determined by a multiplate reader (Lambda Bio-20; Beckman Coulter, Inc., Brea, CA, USA). The cell proliferation assay was carried out six times.

Cell cycle analysis. Transfected OS cells were collected and fixed in $75 \%$ ethanol at $-20^{\circ} \mathrm{C}$ for $16 \mathrm{~h}$. For the cell cycle analysis, the cells were collected, washed twice in cold phosphate-buffered saline (PBS) and stained with propidium iodide (PI) (Invitrogen), then examined with a BD FACSCalibur flow cytometer (BD Biosciences, San Jose, CA, USA). DNA histograms were analyzed with ModFit software (BD Biosciences). The experiments were repeated in triplicate.

Apoptosis analysis. The percentage of apoptotic cells was determined by Annexin V-FITC and PI staining (BD Pharmingen,
San Diego, CA, USA) according to the manufacturer's instructions. The apoptotic morphology was determined by 4,6-diamidino-2-phenylindole (DAPI) staining. Control or miRNA transfected cells were stained with DAPI $(1 \mu \mathrm{g} / \mu \mathrm{l}$; Sigma-Aldrich) to visualize apoptotic cells with fragmented or condensed nuclei. At least five visual fields were observed under a fluorescence microscope for each sample (Nikon Eclipse 80i; Nikon, Tokyo, Japan).

Wound-healing assay. After transfections, the cells were seeded in 6-well plates at $2 \times 10^{5}$ cells/well. After $24 \mathrm{~h}$, the cells were washed with PBS and linear scratch wounds were created using a sterile $200 \mu \mathrm{l}$ pipette tip. The cells were then washed three times with PBS and incubated in DMEM medium containing 5\% FBS. Migration at the wound site was observed under an inverted microscope and images were captured at 0 and $24 \mathrm{~h}$. The percentage of wound closure was analyzed using ImageJ (version 1.44 software; NCBI). Experiments were performed in triplicate.

Cell invasion assay. U2OS cells were transfected with the miR-26b mimics, PFKFB3-siRNA or negative control (NC), cultivated for $24 \mathrm{~h}$, and transferred at the top of Matrigel-coated chambers (24-well insert, $8-\mu \mathrm{m}$ pore size; BD Biosciences) in a serum-free DMEM. Medium containing 10\% fetal calf serum was added to the lower chamber as a chemoattractant. After incubation for $48 \mathrm{~h}$ non-invaded cells were removed from the upper well with cotton swabs while the invaded cells were subjected to H\&E staining, and photographed (magnification, $\mathrm{x} 200$ ) in five independent fields for each well. Each test was repeated in triplicate.

Gelatin zymography. This experiment was conducted to detect MMP-2 and MMP-9 enzyme activity. The medium without the serum was collected from the transfected cells after $24 \mathrm{~h}$. MMP activity was measured by SDS-PAGE under non-reducing conditions (the gel contained $1 \%$ gelatin and $30 \%$ acrylamide). Following electrophoresis, gels were incubated in renaturation buffer $(2.5 \%$ Triton $\mathrm{X}-100$ and $5 \mathrm{mM}$ $\mathrm{CaCl}_{2}$ ) for $30 \mathrm{~min}$ at room temperature. After that the gel was equilibrated in zymogram-developing buffer $(50 \mathrm{mM}$ Tris, $200 \mathrm{mM} \mathrm{NaCl}, 10 \mathrm{mM} \mathrm{CaCl}_{2}, \mathrm{pH} \mathrm{7.5)}$ for $30 \mathrm{~min}$ at room temperature and then incubated at $37^{\circ} \mathrm{C}$ overnight. MMP activity was visualized by staining with Coommasie Blue R-250 (Sigma-Aldrich).

Western blot analysis. Western blotting was performed according to the standard methods as previously described (11) using anti-VEGF, anti-MMP-9, anti-MMP-2, anti-cyclinD1, anti-p27 (Cell Signaling, Danvers, MA, USA), anti-LDHA, anti-GLUT-1 antibodies (Santa Cruz Biotechnology, Inc., Santa Cruz, CA, USA) and anti-PFKFB3 (Proteintech, Chicago, IL, USA). The membranes were stripped and reprobed with anti- $\beta$-actin antibody (Sigma-Aldrich) as a loading control. The experiments were repeated three times.

Measurement of secreted VEGF protein. The level of VEGF in the cell culture supernatant was measured with a commercially available ELISA kit (DVE00; R\&D Systems, Minneapolis, MN, USA) according to the manufacturer's instructions. 
Quantitative RT-PCR. Total RNA and miRNA was extracted using TRIzol reagent (Invitrogen) and miRNeasy Mini kits (Qiagen, Valencia, CA, USA) according to the manufacturer's instructions. Total RNA ( $2 \mu \mathrm{g})$ was reverse transcribed with Omniscript Reverse Transcriptase (Qiagen) in a 20- $\mu l$ reaction mixture using oligo-d(T)12-18 primer. RT-PCR was carried out with the Platinum PCR SuperMix (Invitrogen). Quantitative RT-PCR (RT-qPCR) for PFKFB3 mRNA was performed using a SYBR-Green kit (Roche Diagnostics, Mannheim, Germany) and Light Cycler 480 System II (Roche). The primers used for the specific amplification for human PFKFB3 were: mRNA: 5'-GATGCCCTTCAGGAAAGCCT-3' and 5'-TTGAACACTTTTGTGGGGACGC-3' (NCBI reference sequence: NM_001145443.1) and 18S: 5'-GTAACCCGTTGA ACCCCATT-3' and 5'-CC ATCCAATCGGTAGTAGCG-3' (NCBI reference sequence: M10098). The specificity of each PCR product was assessed by the melting curve analysis and agarose gel electrophoresis. The expression of miR-26b was determined by RT-qPCR using TaqMan MicroRNA Assay kits (Life Technologies, Carlsbad, CA, USA). Expression level of $18 \mathrm{~S}$ was used as internal control for mRNAs, and the U6 level was regarded as an internal miRNA control.

Vectors and luciferase reporter assays. The 3'UTR of human PFKFB3, which contains a miR-26b binding site, was PCR-amplified from genomic DNA and cloned downstream of Renilla luciferase in psiCHECK-2 vector (Promega, Madison, WI, USA). Mutation in 3'UTR of PFKFB3 gene was generated using the QuikChange Site-Directed Mutagenesis kit (Stratagene, Santa Clara, CA, USA). U2OS cells $\left(1 \times 10^{5}\right)$ were plated on 24-well plates. After $24 \mathrm{~h}$, the cells were co-transfected with $100 \mathrm{ng}$ reporter plasmid and $100 \mathrm{nmol} / \mathrm{l}$ of miR-26 mimic or scramble mimic using Lipofectamine 2000. A luciferase reporter construct containing the miR-26b consensus target sequence served as positive control (PC). After $48 \mathrm{~h}$, the cells were lysed using passive lysis buffer and luciferase activities were measured with the Dual-Luciferase Assay kit (Promega). Renilla luciferase signal was normalized to the firefly luciferase signal. Three independent experiments were performed in triplicate.

Measurement of lactate and ATP production. U2OS cells were treated with or without miR-26b or scramble mimics $(100 \mathrm{~nm})$ in serum-free medium for $24 \mathrm{~h}$. Culture medium $(50 \mathrm{ml})$ was collected from each sample and measured for lactate concentration using a lactate ELISA kit (BioVision, Mountain View, CA, USA) according to the manufacturer's instructions. While intracellular levels of ATP in the indicated groups were measured using s CellTiter-Glo ${ }^{\circledR}$ Luminescent Assay (Promega), the values were normalized to the scrambled control.

Measurement of glucose uptake, oxygen consumption and mitochondrial membrane potential (MMP). For glucose uptake, $1 \times 10^{5}$ cells were stained with fluorescent glucose analogue 6-( $N$-(7-nitrobenz-2-oxa-1,3-diazol-4-yl)amino)2-deoxyglucose (6-NBDG; $50 \mu \mathrm{M}$; Invitrogen) for $20 \mathrm{~min}$, washed with PBS and analyzed by flow cytometry. $\mathrm{O}_{2}$ consumption was measured using an XF24 Extracellular Flux Analyzer (Seahorse Biosciences, Billerica, MA, USA) as per the manufacturer's instructions. The experiments were repeated three times in triplicate. The data were expressed in $\mathrm{pMol} / \mathrm{min}$. Flow cytometry was utilized to measure alterations in MMP using cationic fluorescent dye 1,1',3,3'-tetraethylbenzamidazolocarbocyanin iodide (JC-1; Invitrogen). After completion of the indicated treatment time points, the cells were loaded with $2 \mu \mathrm{M} \mathrm{JC}-1$ in $\mathrm{HBSS}$ at $37^{\circ} \mathrm{C}$ for $15 \mathrm{~min}$. The mean ratios of red vs. green fluorescent signal intensities were measured in three independent experiments.

Statistical analysis. Results were presented as the means \pm SD (standard deviation) unless otherwise specified. Statistical analyses were performed by one-way ANOVA followed by Newman-Keuls post-hoc comparison test. $\mathrm{P} \leq 0.05$ was considered to indicate statistically significant differences (GraphPad Prism 5; GraphPad software, Inc., San Diego, CA, USA).

\section{Results}

Induced expression of miR-26b inhibited OS cell proliferation and cell cycle progression. Findings of a recent study demonstrated significant downregulation of miR-26b in human OS samples (6). However, the effect of miR-26b on OS has not been fully elucidated. Thus, we examined the mechanistic role of miR-26b in OS cells. We investigated the functional significance of miR-26b on growth, cell cycle progression and apoptosis in U2OS cells using miRNA mimics or scramble mimics. Our data showed a significant increase of miR-26b levels ( $\sim 85$-folds) in miR-26b-transfected cells compared to that of scramble or parental cells $(\mathrm{P}<0.001)$ (Fig. 1A). Then we compared the proliferation and cell cycle changes after miR-26b overexpression in OS cells. Our results showed that the ectopic expression of miR-26b suppressed cell proliferation in a timedependent manner $(\mathrm{P}<0.05)$ with a subsequent elevation of the percentage of G1-phase cells and reduction of S-phase cells (by $20 \% \pm 5)(\mathrm{P}<0.01)$, respectively (Fig. 1B and C). Furthermore, treatment with miR-26b resulted in the increased induction of apoptotic cells as demonstrated by Annexin V-FITC Apoptosis Detection kit $(\mathrm{P}<0.01)$ (Fig. 1D). In addition miR-26b mimic enhanced chromatin condensation and the formation of apoptotic bodies identifiable by staining cells with Hoechst 3342 fluorescent dye (Fig. 1E). Taken together, these results indicated that miR-26b efficiently inhibited cell proliferation and cell cycle progression, and induced apoptosis in vitro, thus manifesting the tumor suppressive role of miR-26b in OS cells.

Effect of miR-26b on the migration and invasion of OS cells. miR-26b has been reported to be closely associated with invasiveness and metastasis in malignancy (15). Therefore, we studied the effects of miR-26b in OS cell migration and invasion. We found that both invading and migrating miR-26b overexpressed U2OS cells were greatly decreased (Fig. 2A and B) allowing only $40-50 \%$ of cells to migrate or invade respectively, compared to those with scrambled or untreated cells $(>80 \%)$. Since MMP-2 and MMP-9 play a critical role in tumor cell invasiveness (16), we examined the effect of miR-26b on the enzyme activities of MMP-2 and MMP-9 by employing gelatin zymography. The gelatinolytic activities of MMP-2 and MMP-9 were found to be reduced in conditioned media of U2OS cells, suggesting a 
A
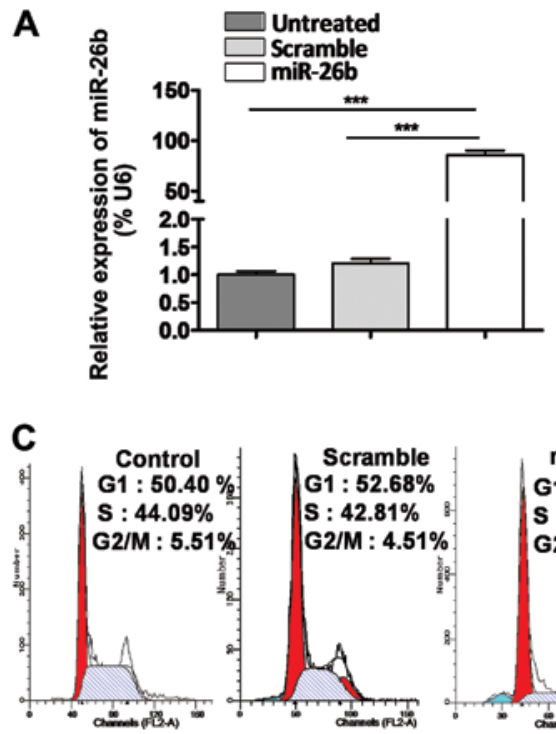

D

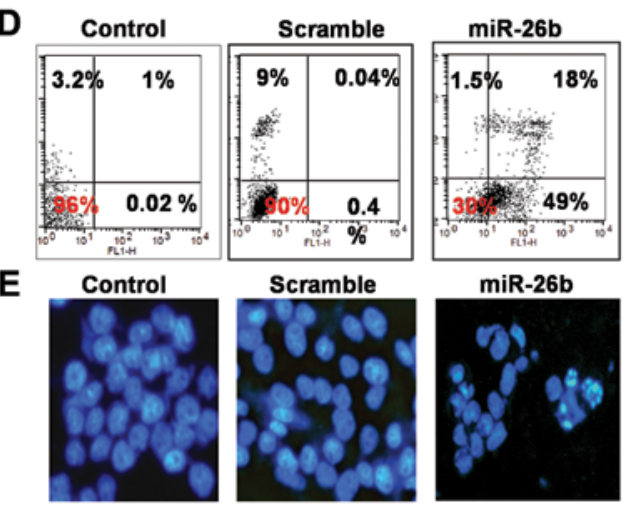

B
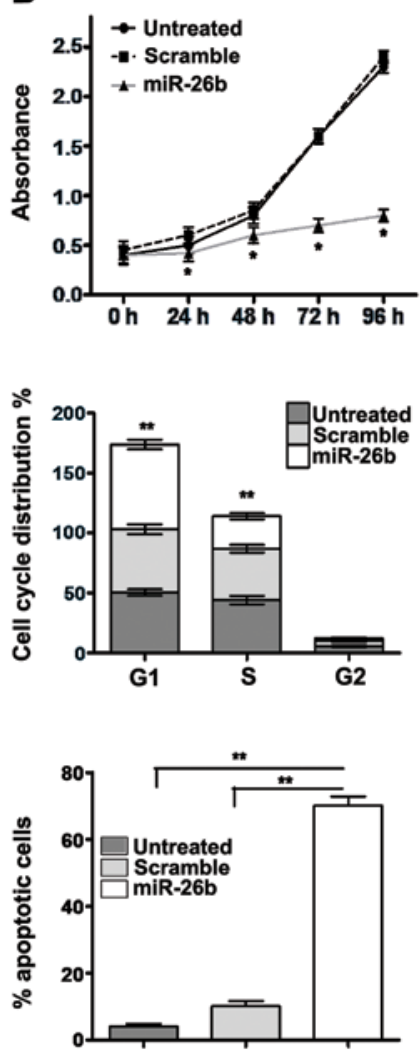

Figure 1. Role of miR-26b in cell proliferation, cell cycle progression and apoptosis of human osteosarcoma (OS) cell line. (A) Expression levels of miR-26b detected in U2OS cells following transfection with miR-26b or scramble mimics, by RT-qPCR. miR-26b expression was significantly increased in cells transfected with miR-26b mimics. (B) CCK-8 assay was performed to examine U2OS cell proliferation following transfection with miR-26b mimics or scramble mimics. Ectopic expression of miR-26b significantly inhibited U2OS cell proliferation in $24,48,72$ and 96 h compared with cells expressing scramble mimics. (C) Cell cycle distribution in U2OS cells following transfection with miR-26b or scramble mimics. miR-26b caused cell cycle arrest at the G0/G1 phase. (D) Overexpression of miR-26b in the U2OS OS cell line induced apoptosis. Cells were stained with the Annexin V-FITC apoptosis detection kit. (E) miR-26b-induced apoptotic body formation in OS cells was determined by DAPI staining and photographed under a fluorescence microscope (x200) as described in Materials and methods. Data are presented as the means \pm SD and are representative of three independent experiments. ${ }^{* *} \mathrm{P}<0.01$ and ${ }^{* * *} \mathrm{P}<0.001$.

reduction in cell invasion by overexpressed miR-26b (Fig. 2C). Simultaneously, we investigated VEGF protein expression levels and found that the miR-26b-transfected group significantly downregulated VEGF expression and VEGF secretion in U2OS conditioned media compared to that of scrambled or untreated groups $(\mathrm{P}<0.05)$ (Fig. 2D and $\mathrm{E})$.

miRNA-26b targeted PFKFB3 and suppressed the expression of LDH-A, GLUT-1, MMP-9, MMP-2 and cyclin D1. To determine the molecular mechanism of tumor progression triggered by miR-26b in OS, putative target genes of miR-26b were searched using Targetscan 6.2. PFKFB3 was predicted to be the target of miR-26b (Fig. 3A). The luciferase assay showed that miR-26b significantly suppressed wild-type (WT) 3'UTR ( $\sim 5$-fold) but not the mutant (MUT) 3'UTR of PFKFB3 reporter activity in $\mathrm{U} 2 \mathrm{OS}$ cells $(\mathrm{P}<0.01)$ (Fig. 3B). In addition, overexpression of miR-26b downregulated PFKFB3 mRNA and protein levels $(\mathrm{P}<0.01)$, suggesting the post-transcriptional regulation of $P F K F B 3$ gene expression by miR-26b (Fig. 3C and D). To examine the mechanism of how miR-26b influences the glycolysis and invasive phenotype of OS cells, we measured the expression of LDHA, GLUT-1, MMP-9 and MMP-2 using western blot analysis. The results showed that overexpression of miR-26b decreased the expression of LDH-A, GLUT-1, MMP-9 and MMP-2 in U2OS cells. Moreover, the expression of G1-S regulatory proteins was analyzed demonstrating G0/G1 cell cycle arrest by downregulation of cyclin D1 and elevation of p27 levels in the same experimental conditions.

Ectopic expression of miR-26b inhibits glycolytic phenotype in OS cells. Induction of aerobic glycolysis in OS cells accomplishes survival advantage and invasiveness accompanied by enhanced glucose uptake, glycolytic flux into lactate and reduced mitochondrial function $(11,17)$. miRNAs were shown to closely regulate cancer-associated glycolytic pathways (18). Our findings showed that miR-26b mediated the significant downregulation of PFKFB3 levels, the enzyme 

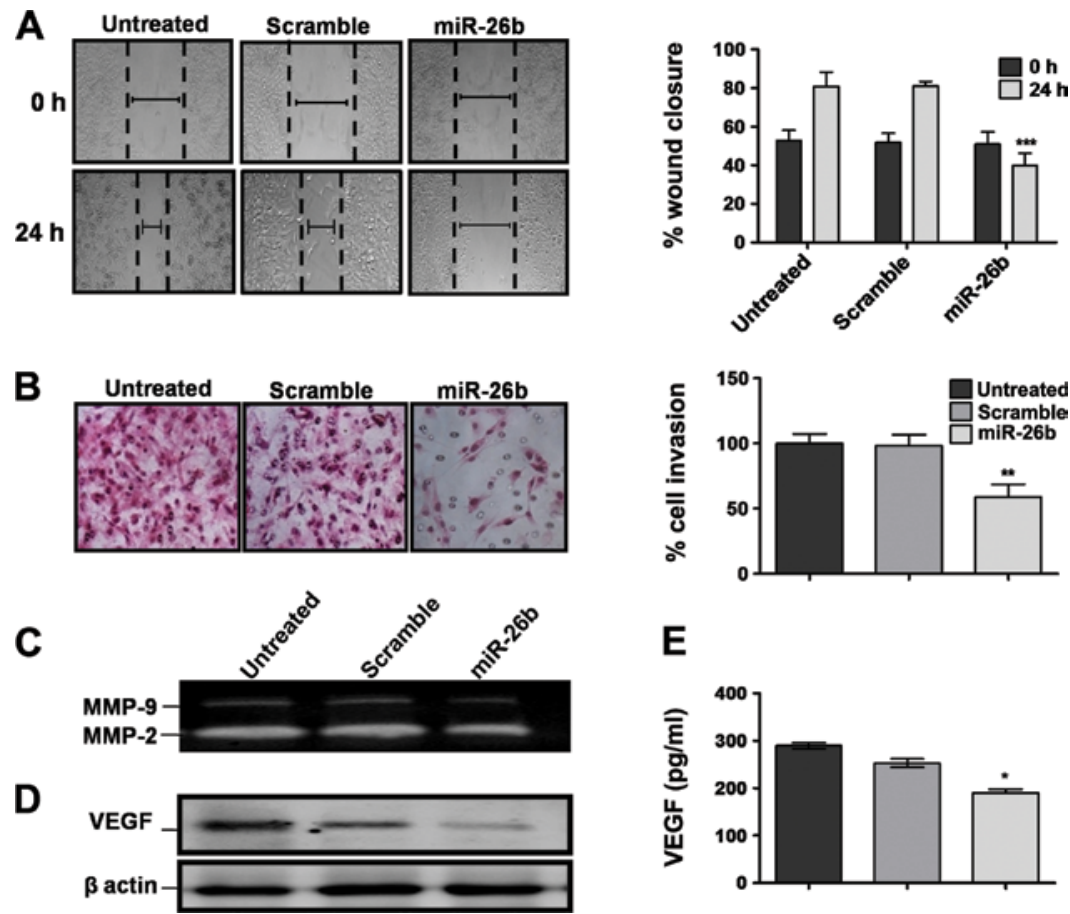

Figure 2. Effect of miR-26b on the migration and invasion ability of osteosarcoma cells. (A) Scratch wound-healing assay was conducted in the control, scrambled or miR-26b mimics-transfected U2OS cells. Cell migration was measured at 0 and $24 \mathrm{~h}$ after cells were scratched. (B) U2OS cells expressing scramble or miR-26b were plated on Matrigel-coated Transwell chambers and analyzed for cell invasion after $24 \mathrm{~h}$ as described in the experimental procedures. Data are presented as the percentage wound closure and of cell invasion. (C) MMP-2 and MMP-9 expression in U2OS cells was assessed by gelatin zymography analysis $24 \mathrm{~h}$ after the indicated transfections. (D) Western blot analysis of VEGF expression in scrambled or miR-26b-transfected cells. $\beta$-actin was used as the loading control. (E) Conditioned media were collected $24 \mathrm{~h}$ after the indicated transfections and analyzed for the secreted VEGF (pg/ml) levels by ELISA. Values are the means $\pm \mathrm{SD}(\mathrm{n}=3) ;{ }^{*} \mathrm{P}<0.05$ and ${ }^{* *} \mathrm{P}<0.01$.

A

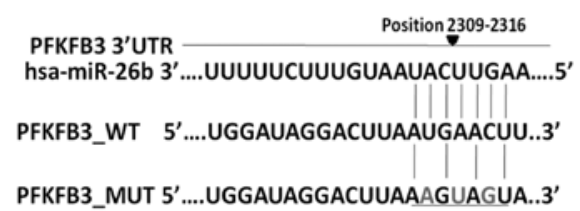

C

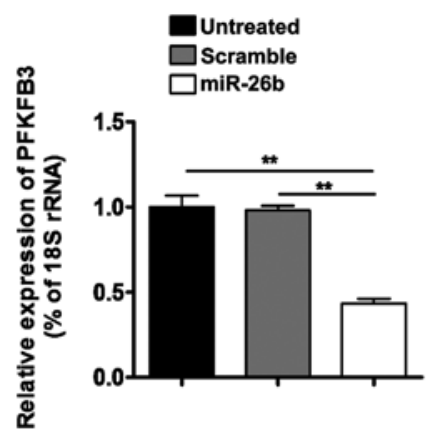

B

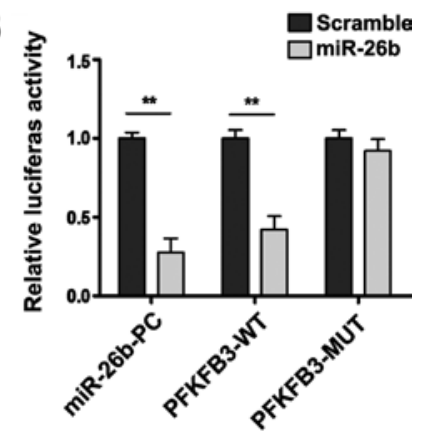

D

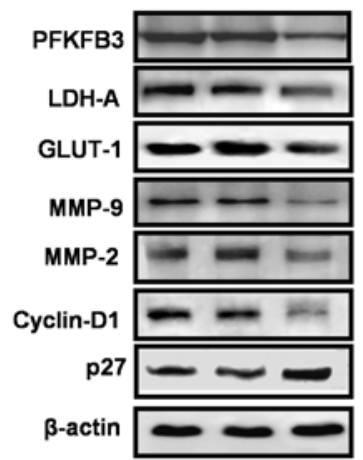

Figure 3. miR-26b negatively regulates $P F K F B 3$ gene in osteosarcoma cells. (A) Computational analysis showed that miR-26b potentially targeted the $P F K F B 3$ gene. Schematic presentation of predicted PFKFB3 3'UTR as target site for miR-26b based on Targetscan 6.2. Site-directed mutagenesis was performed to mutate base pairs in the predicted seed region targeted by miR-26b in the PFKFB3 3'UTR. The mutant binding site is underlined. (B) U2OS cells were co-transfected with a luciferase reporter plasmid encoding the wild-type (WT) or mutated (MUT) 3'UTR of human PFKFB3 along with miR-26b mimics. After $24 \mathrm{~h}$ of transfection, luciferase activity was measured. (C) The RT-qPCR assay was performed to detect mRNA expression of PFKFB3 on transfection with miR-26b or scramble mimics. Ectopic expression of miR-26b downregulated the mRNA level of PFKFB3. (D) U2OS cells transfected with miR-26b mimics or scramble mimics were subjected to western blot analysis. Blots indicating the protein expression of PFKFB3 and its relative downstream proteins which are known to regulate glycolytic flux, invasion and cell cycle progression in cancer cells. Experiments were repeated three times. Error bars indicate the means $\pm \mathrm{SD}$ of three independent experiments. ${ }^{* *} \mathrm{P}<0.01$. 
A

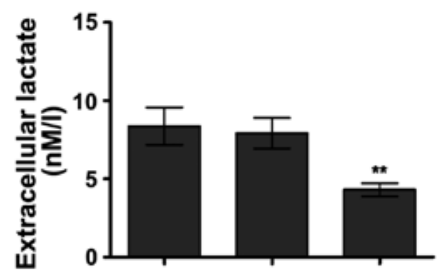

C

E
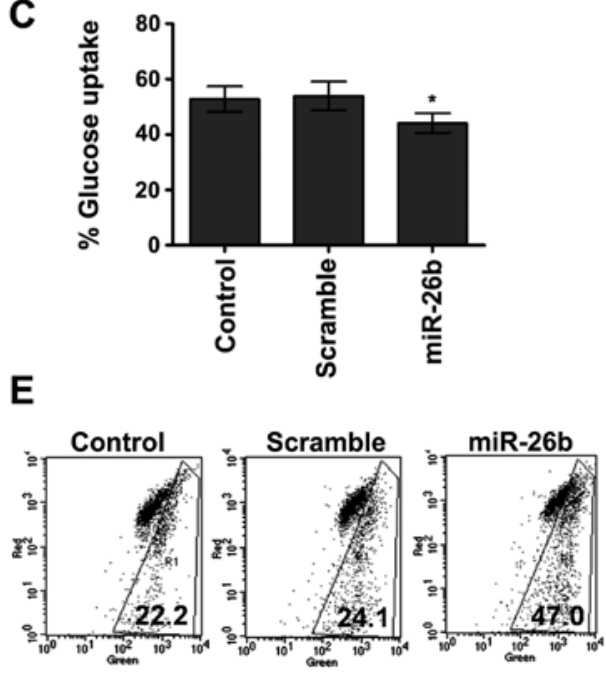

B

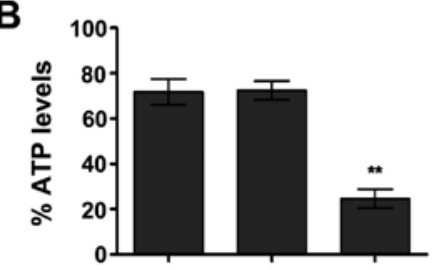

D
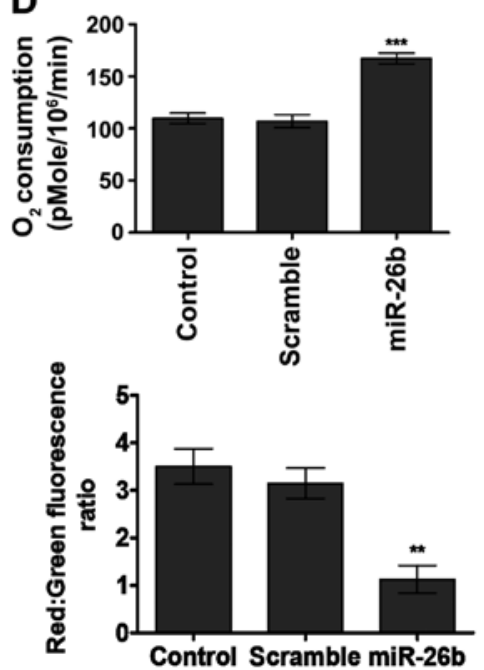

Figure 4. Effect of miR-26b on the glycolytic phenotype in osteosarcoma (OS) cells. Re-expression of miR-26b inhibits glycolytic flux to lactate, ATP and glucose uptake with a simultaneous increase in the oxygen consumption rate. (A) U2OS cells treated with or without miR-26b mimics were subjected to the measurement of glycolytic activity. Extracellular lactate was measured by an NADH-coupled enzyme reaction and expressed as nmol/ $\mu 1$ for each transfected group. (B) Intracellular ATP concentrations were measured by an ATP luciferase assay and expressed as a percentage of the untreated controls. The ATP level of untreated cells at time t, 0 was set to $100 \%$. The lactate and ATP levels were suppressed in response to miR-26b overexpression. (C) Graph depicting increased oxygen consumption (pmol $\mathrm{O}_{2} / 10^{6} / \mathrm{min}$ ) in cells ectopically expressing miR-26b using XF24 extracellular flux analyzer. (D) Glucose uptake was quantified in the indicated transfected groups through flow cytometry using the glucose analogue 6-NBDG. (E) Mitochondrial membrane potential in the indicated transfected groups was quantified through flow cytometry using the fluorescence dye JC-1. The column bar graphs show a decrease in the red:green fluorescence ratio in response to miR-26b mimics. Representative data of three independent experiments are presented. ${ }^{* *} \mathrm{P}<0.01$ and ${ }^{* * *} \mathrm{P}<0.001$.

of which is known to regulate high glycolytic flux in cancer cells (19). Therefore, we observed the effects of miR-26b on various glycolytic parameters. The results indicated that cell expressing miR-26b mimics induced a decrease in extracellular lactate $(\mathrm{P}<0.01)$, ATP levels $(\mathrm{P}<0.01)$ and glucose consumption $(\mathrm{P}<0.05)$ in U2OS cells (Fig. 4A-C). Furthermore, suppression of the glycolytic phenotype was accompanied by a metabolic shift towards mitochondrial respiration indicated by an increase in the oxygen consumption rate $(\mathrm{P}<0.001)$ (Fig. 4D). In addition, our results showed a significant decrease in the mitochondrial membrane potential in cells overexpressing miR-26b compared to that of scramble or untreated cells $(\mathrm{P}<0.01)$ (Fig. 4E). Taken together, these results suggested that miR-26b the induced metabolic shift away from the glycolytic pathway and thereby may be responsible for the suppression of migration and invasion in OS cells.

PFKFB3 knockdown inhibited cell proliferation and cell cycle progression in OS cells. The tumor progressive role of PFKFB3 has been demonstrated in several types of cancer by elevation of the high glycolytic rate (19). Our results have shown miR-26b arbitrated antitumor and anti-metabolic effects by potent inhibition of the PFKFB3 gene with subsequent inhibition of the glycolytic pathway. However, the growth inhibitory effects mediated by silencing of PFKFB3 in OS have not been studied previously. In line of these observations, we hypothesized that the direct suppression of PFKFB3 may have an inhibitory effect on cell population growth in OS cells. To confirm, we silenced the expression of PFKFB3 by RNA interference directed against the PFKFB3 gene (Fig. 5A). Results showed that inhibition of PFKFB3 expression markedly arrested cell cycle progression (Fig. 5B), reduced cell proliferation (Fig. 5C) and induced apoptosis (Fig. 5D), accompanied by impaired cell migration (Fig. 5E) and cell invasion (Fig. 5F). Taken together, these findings suggested that PFKFB3 induces OS progression and aggressiveness. Overexpression of miR-26b mediated suppression of cell proliferation and invasion were partially attributed to the inhibition of $P F K F B 3$ gene expression.

\section{Discussion}

miRNAs are short non-coding RNAs that post-transcriptionally modify gene expression in eukaryotic cells. Expression of single miRNA modulates many cell functions by regulating the expression of a wide array of genes (20). The differential expression of miRNA between normal and tumor tissues has been previously identified. This emphasizes the tumor-promoting role of dysregulated miRNA expression in cancer cells (20). Therefore, understanding the functional 

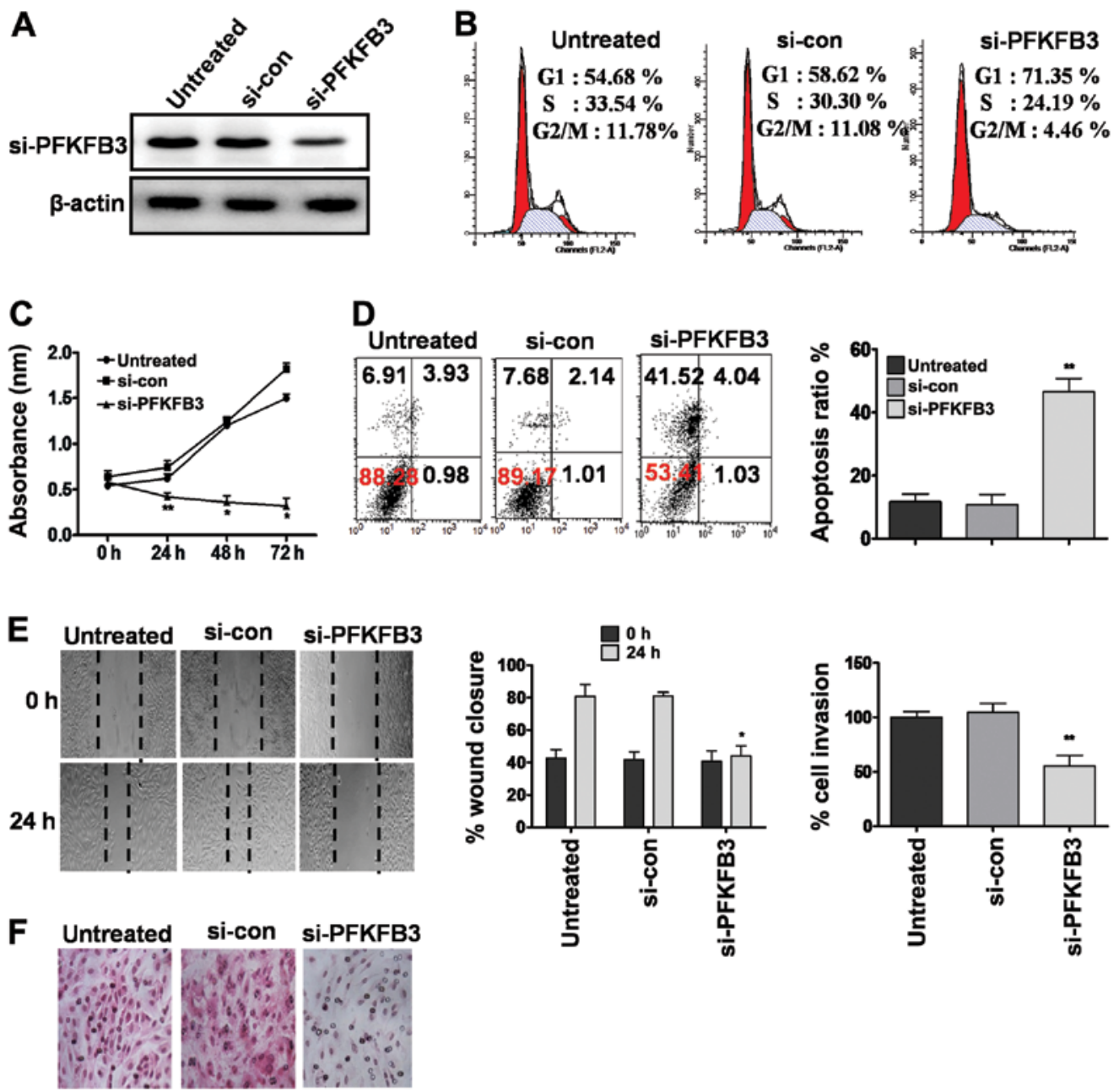

Figure 5. Effects of si-PFKFB3 on the osteosarcoma cell cycle progression, cell proliferation, apoptosis, cell migration and invasion. (A) Western blot analysis was performed to confirm the PFKFB3 knockdown after $24 \mathrm{~h}$ of transfection with si-control or si-PFKFB3 in U2OS cells. (B) Cell cycle analysis was performed in si-RNA-transfected cells by propidium iodide staining. Suppression of PFKFB3 arrested the cell cycle in G1 phase. (C) Cell proliferation was determined in si-control or si-PFKFB3-transfected cells using CCK-8. Depletion of PFKFB3 inhibited cell proliferation. (D) Induction of apoptosis in PFKFB3 silenced cells was detected by flow cytometric analysis as described in Materials and methods. (E) The wound-healing assay was performed to determine the effect of PFKFB3 suppression on U2OS cell migration. Data are presented as the percentage of wound closure. Downregulation of PFKFB3 inhibited cell migration. (F) Transwell invasion assay was performed to analyze the percentage of cell invasion in response to PFKFB3 silencing. Graph shows a decrease in cell invasive ability in si-PFKFB3-transfected cells. Experiments were repeated three times. Error bars indicate the means \pm SD of three independent experiments. ${ }^{*} \mathrm{P}<0.05$ and ${ }^{* *} \mathrm{P}<0.01$.

mechanism of miRNAs by identification of its putative molecular targets is important.

A recent study has demonstrated the miRNA expression signature associated with OS-characterizing pathogenesis, clinical metastasis and chemotherapy response (6). Expression profiling from 18 pretreated OS tumors were compared to 12 normal bone tissues. RT-qPCR confirmed differential expression of miRNAs with substantial downregulation of miR-26b in OS patient samples compared to healthy individuals (6). However, the precise molecular mechanism mediated by miR-26b in OS remains unclear. To the best of our knowledge, in the present study, we performed for the first time functional assays to provide insight into the biological role played by miR-26b in OS. Few recent studies have reported the miR-26b downregulation in other types of cancer including breast cancer (15), hepatocellular (21), nasopharyngeal (8) and lung carcinoma, and glioma $(22,23)$. Mechanistically, miR-26b has shown to be tumor suppressive by modulating a diverse array of cellular and molecular responses in cancer cells. The putative molecular targets of miR-26b-mediated antiproliferative and apoptogenic response includes NFkB, USP9X in HCC $(24,25)$, EphA2 in glioma (23), PTGS2, SLC7A11 in breast cancer $(26,27)$ and SODD in melanoma $(28)$.

We have shown that reinstating the expression of miR-26b inhibited OS cell proliferation, cell cycle progression and apoptosis induction. OS encounters treatment failure due to metastasis and chemoresistance, while the underlying mechanism remains unclear. Therefore, we investigated the role of miR-26b on OS cell migration and invasion. The results showed that overexpressed miR-26b significantly suppressed the migration and invasive ability of U2OS cells in vitro. Previous studies have demonstrated the enhanced expression of matrix metalloproteinases (MMPs) such as MMP-2 and MMP-9 in promoting OS cell metastasis by degrading components of the basement membrane and epimatrix (16). In addition, an increased expression of vascular endothelial growth factor 
(VEGF) has been associated with increasing malignant potential of OS cells (29). We found that the ectopic expression of miR-26b significantly downregulated MMP-2 and MMP-9 enzymatic activities and VEGF expression levels. Thus, our results suggest the tumor suppressive role of miR-26b, which concurs with other studies (23-26) and provided a basis for identification of a promising therapeutic agent for OS.

Few recent studies have demonstrated the unique defects in mitochondrial oxidative phosphorylation in OS cells resulting in metabolic shift towards the glycolytic pathway (11). The high prevalence of glycolysis and dysregulation of oxidative metabolism, known as the Warburg effect, provides survival advantage and aggressive phenotype in OS cells $(11,17,30)$. Moreover, the administration of 2-deoxy-d-glucoe (2-DG), a potent glycolytic inhibitor has been shown to sensitize OS cell growth and bioenergetic functions demonstrated by reduced cell viability and intracellular ATP levels, indicating glycolytic dependency of OS cells (31). Another study has demonstrated the anti-metastatic effects of glycolytic inhibition via 2-DG in the highly metastatic DLM8-luc-M1 OS cell line accompanied by cytoskeletal rearrangements and inhibition of cathepsin expression (17). Therefore, the renewed interest to characterize the metabolic vulnerabilities in OS has led to examination of the underlying genes including their products and microRNAs, which may provide a novel avenue for metastatic prevention.

In line of these evidences, we identified PFKFB-3 as a direct functional target of miR-26b using a prediction program. Computational analysis revealed binding sites for the miR-26b seed sequence at 3'UTR of PFKFB3. Furthermore, reinstatement of miR-26b expression led to the decrease in luciferase activity of wild-type PFKFB3 3'UTR in U2OS cells whereas the site-directed mutation attenuated miR-26b regulation. In addition, results from the RT-qPCR and protein expression analysis indicated that the ectopic expression of miR-26b suppressed mRNA and protein levels of PFKFB3 simultaneously. Taken together, these results suggest that miR-26b regulated the expression of PFKFB3 by directly targeting 3'UTR of PFKFB3 in OS cells.

PFKFB3 is a key regulator of high glycolytic flux in various types of cancer compared to normal tissues (19), regulated by HIF1 $\alpha$, PTEN and Akt (32-34). Notably, PFKFB3 has drawn particular interest in the pharmaceutical industry since mRNA and protein levels of PFKFB3 are upregulated in most tumor types (19). PFKFB3 regulates the synthesis of fructose 2,6-bisphosphate (F26BP) which is an activator of 6-phosphofructo-1-kinase (PFK-1), a key step of glycolysis. Notably, genomic deletion of PFKFB3 suppressed glucose metabolism and tumor growth in vitro and in vivo (35) rendering this enzyme a potential target for anticancer therapy. Previously a small molecule competitive inhibitor of PFKFB3 was identified, known as 3-(3-pyridinyl)-1-(4-pyridinyl)2-propen-1-one (3PO). 3PO induced the suppression of glucose metabolism and antitumor activity in several human cancer cell lines in vitro and xenograft tumors in vivo (36) but the efficacy of $3 \mathrm{PO}$ was compromised due to metabolic resistance and its pharmacokinetic profile, which renders it inappropriate for evaluation in human subjects. Recently, the functional involvement of PFKFB3 in the regulation of CDK1 activity has been shown where siRNA-mediated silencing of PFKFB3 caused cell cycle arrest at G0/G1 accompanied by increased apoptosis in HeLa cells (37). These studies signify that re-establishment for the development of novel molecular inhibitors of PFKFB3 is necessary. miRNA-mediated regulation of altered metabolic pathways in various types of cancer has been identified (18). Emerging evidence has proven that miRNAs are significant regulators of glucose metabolism although miRNA predictions have yet to be verified. Based on microarray- and NGS-comprised validation methods, miRNAs including hsa-miR-26b-5p and hsa-miR-330-3p are predicted to obtain putative binding sites at 3'UTR of PFKFB3 (Linkouts: TargetScan 5.1, MicroCosm, microRNAMap 2.0) although the functional validation remained to be investigated.

As demonstrated, PFKFB3 is a crucial component of aerobic glycolysis in cancer cells (38) including OS (14). We investigated the effects of miR-26b on molecules modulating glycolysis, invasion and cell cycle progression in OS cells. The results showed that the overexpression of miR-26b significantly downregulated the protein expression of PFKFB3, lactate dehydrogenase (LDHA), glucose transporter (GLUT-1), MMP-9, MMP-2 and cyclin D1 while p27 levels were upregulated. The results were consistent with the decrease of glycolytic phenotype demonstrated by a decrease in extracellular lactate, ATP levels and glucose consumption in OS cells. By contrast, we observed an elevated oxygen consumption rate accompanied by loss of mitochondrial membrane potential. These results indicate that miR-26b substantially downregulated glycolytic activity but enhanced mitochondrial respiration without a concominant increase in energy production suggesting participation of miR-26b in the possession of glycolytic dependency by OS cells. In addition, antitumor effects induced by miR-26b were mediated through the downregulation of PFKFB3 permitting the inactivation of the glycolytic pathway.

In summary, our findings have demonstrated the tumor suppressive effects of miR-26b in OS cells through suppression of the glycolytic pathway by targeting PFKFB3 gene. However, restoration of miR-26b decreased the expression of LDHA, GLUT1, MMP-9, MMP-2 and cyclin D1 leading to the attenuation of glycolytic activity, cell proliferation, migration, invasion and apoptosis induction in vitro. In addition, genomic inhibition of PFKFB3 alone induced significant antiproliferative effects in OS cells. These results show a new approach in the molecular therapy of OS by the overexpression of miR-26b, having the potential to manifest substantial tumor suppressive effects by targeting altered metabolism in cancer cells. The findings suggest further investigation of the miR-26b as a promising biomarker and therapeutic target in OS treatment.

\section{Acknowledgements}

This study was supported by the Science and Technology Planning Project of Zhejiang Province (no. 2012B031834032), and the Zhejiang Natural Science Foundation (no. S24230400143).

\section{References}

1. Geller DS and Gorlick R: Osteosarcoma: a review of diagnosis, management, and treatment strategies. Clin Adv Hematol Oncol 8: 705-718, 2010.

2. Ottaviani G and Jaffe N: The epidemiology of osteosarcoma. Cancer Treat Res 152: 3-13, 2009. 
3. Marina N, Gebhardt M, Teot L and Gorlick R: Biology and therapeutic advances for pediatric osteosarcoma. Oncologist 9: 422-441, 2004

4. Wang Z, Cai H, Lin L, Tang M and Cai H: Upregulated expression of microRNA-214 is linked to tumor progression and adverse prognosis in pediatric osteosarcoma. Pediatr Blood Cancer 61 : 206-210, 2014

5. Ambros V: MicroRNA pathways in flies and worms: growth, death, fat, stress, and timing. Cell 113: 673-676, 2003.

6. Jones KB, Salah Z, Del Mare S, et al: miRNA signatures associate with pathogenesis and progression of osteosarcoma Cancer Res 72: 1865-1877, 2012.

7. Li J, Li X, Kong X, Luo Q, Zhang J and Fang L: MiRNA-26b inhibits cellular proliferation by targeting CDK8 in breast cancer. Int J Clin Exp Med 7: 558-565, 2014.

8. Ji Y, He Y, Liu L and Zhong X: MiRNA-26b regulates the expression of cyclooxygenase-2 in desferrioxamine-treated CNE cells FEBS Lett 584: 961-967, 2010

9. Zhang C, Tong J and Huang G: Nicotinamide phosphoribosyl transferase (Nampt) is a target of microRNA-26b in colorectal cancer cells. PLoS One 8: e69963, 2013.

10. Gramantieri L, Fornari F, Callegari E, et al: MicroRNA involvement in hepatocellular carcinoma. J Cell Mol Med 12: 2189-2204, 2008.

11. Shapovalov Y, Hoffman D, Zuch D, de Mesy Bentley KL and Eliseev RA: Mitochondrial dysfunction in cancer cells due to aberrant mitochondrial replication. J Biol Chem 286 22331-22338, 2011.

12. Giang AH, Raymond T, Brookes P, et al: Mitochondrial dysfunction and permeability transition in osteosarcoma cells showing the Warburg effect. J Biol Chem 288: 33303-33311, 2013.

13. Van Schaftingen E, Hue L and Hers HG: Fructose 2,6-bisphosphate, the probably structure of the glucose- and glucagon-sensitive stimulator of phosphofructokinase. Biochem J 192: 897-901, 1980.

14. Zawacka-Pankau J, Grinkevich VV, Hünten S, et al: Inhibition of glycolytic enzymes mediated by pharmacologically activated p53: targeting Warburg effect to fight cancer. J Biol Chem 286: 41600-41615, 2011.

15. Verghese ET, Drury R, Green CA, et al: MiR-26b is downregulated in carcinoma-associated fibroblasts from ER-positive breast cancers leading to enhanced cell migration and invasion. J Pathol 231: 388-399, 2013.

16. Liao CL, Lin JH, Lien JC, et al: The crude extract of Corni Fructus inhibits the migration and invasion of U-2 OS human osteosarcoma cells through the inhibition of matrix metalloproteinase-2/-9 by MAPK signaling. Environ Toxicol: Aug 19, 2013 (Epub ahead of print). doi: 10.1002/tox.21894.

17. Sottnik JL, Lori JC, Rose BJ and Thamm DH: Glycolysis inhibition by 2-deoxy-D-glucose reverts the metastatic phenotype in vitro and in vivo. Clin Exp Metastasis 28: 865-875, 2011

18. Singh PK, Mehla K, Hollingsworth MA and Johnson KR: Regulation of aerobic glycolysis by microRNAs in cancer. Mol Cell Pharmacol 3: 125-134, 2011.

19. Atsumi T, Chesney J, Metz C, et al: High expression of inducible 6-phosphofructo-2-kinase/fructose-2,6-bisphosphatase (iPFK-2 PFKFB3) in human cancers. Cancer Res 62: 5881-5887, 2002.

20. Tutar Y: miRNA and cancer; computational and experimental approaches. Curr Pharm Biotechnol 15: 429, 2014.

21. Ji J, Shi J, Budhu A, et al: MicroRNA expression, survival, and response to interferon in liver cancer. N Engl J Med 361: 1437-1447, 2009.
22. Arora H, Qureshi R, Park AK and Park WY: Coordinated regulation of ATF 2 by miR-26b in $\gamma$-irradiated lung cancer cells. PLoS One 6: e23802, 2011.

23. Wu N, Zhao X, Liu M, et al: Role of microRNA-26b in glioma development and its mediated regulation on EphA2. PLoS One 6: e16264, 2011.

24. Zhao N, Wang R, Zhou L, Zhu Y, Gong J and Zhuang SM: MicroRNA-26b suppresses the NF- $\mathrm{KB}$ signaling and enhances the chemosensitivity of hepatocellular carcinoma cells by targeting TAK1 and TAB3. Mol Cancer 13: 35, 2014.

25. Shen G, Lin Y, Yang X, Zhang J, Xu Z and Jia H: MicroRNA-26b inhibits epithelial-mesenchymal transition in hepatocellular carcinoma by targeting USP9X. BMC Cancer 14: 393, 2014

26. Li J, Kong X, Zhang J, Luo Q, Li X and Fang L: MiRNA-26b inhibits proliferation by targeting PTGS2 in breast cancer. Cancer Cell Int 13: 7, 2013.

27. Liu XX, Li XJ,Zhang B, et al: MicroRNA-26b is underexpressed in human breast cancer and induces cell apoptosis by targeting SLC7A11. FEBS Lett 585: 1363-1367, 2011

28. Zhang B, Liu XX, He JR, et al: Pathologically decreased miR-26a antagonizes apoptosis and facilitates carcinogenesis by targeting MTDH and EZH2 in breast cancer. Carcinogenesis 32: 2-9, 2011.

29. Ohba T, Cates JM, Cole HA, et al: Autocrine VEGF/VEGFR1 signaling in a subpopulation of cells associates with aggressive osteosarcoma. Mol Cancer Res 12: 1100-1111, 2014.

30. Palorini R, Votta G, Balestrieri C, et al: Energy metabolism characterization of a novel cancer stem cell-like line 3AB-OS. J Cell Biochem 115: 368-379, 2014.

31. Issaq SH, Teicher BA and Monks A: Bioenergetic properties of human sarcoma cells help define sensitivity to metabolic inhibitors. Cell Cycle 13: 1152-1161, 2014.

32. Minchenko A, Leshchinsky I, Opentanova I, et al: Hypoxiainducible factor-1-mediated expression of the 6-phosphofructo2-kinase/fructose-2,6-bisphosphatase-3 (PFKFB3) gene. Its possible role in the Warburg effect. J Biol Chem 277: 6183-6187, 2002.

33. Garcia-Cao I, Song MS, Hobbs RM, et al: Systemic elevation of PTEN induces a tumor-suppressive metabolic state. Cell 149: 49-62, 2012.

34. Manes NP and El-Maghrabi MR: The kinase activity of human brain 6-phosphofructo-2-kinase/fructose-2,6-bisphosphatase is regulated via inhibition by phosphoenolpyruvate. Arch Biochem Biophys 438: 125-136, 2005.

35. Telang S, Yalcin A, Clem AL, et al: Ras transformation requires metabolic control by 6-phosphofructo-2-kinase. Oncogene 25: 7225-7234, 2006

36. Clem B, Telang S, Clem A, et al: Small-molecule inhibition of 6-phosphofructo-2-kinase activity suppresses glycolytic flux and tumor growth. Mol Cancer Ther 7: 110-120, 2008.

37. Yalcin A, Clem BF, Imbert-Fernandez Y, et al: 6-Phosphofructo2-kinase (PFKFB3) promotes cell cycle progression and suppresses apoptosis via Cdk1-mediated phosphorylation of p27. Cell Death Dis 5: e1337, 2014.

38. Almeida A, Bolaños JP and Moncada S: E3 ubiquitin ligase APC/C-Cdh1 accounts for the Warburg effect by linking glycolysis to cell proliferation. Proc Natl Acad Sci USA 107: 738-741, 2010 . 